\title{
CRESCIMENTO DE CRIANÇAS EM ALEITAMENTO MATERNO EXCLUSIVO NO PRIMEIRO SEMESTRE DE VIDA
}

\author{
GROWTH OF CHILDREN IN EXCLUSIVE BREASTFEEDING IN THEIR \\ FIRST SEMESTER OF LIFE
}

Rosângela Aparecida Augusto*

José Maria Pacheco de Souza**

\begin{abstract}
Augusto RA, Souza JMP. Crescimento de crianças em aleitamento materno exclusivo no primeiro semestre de vida. Rev Bras Crescimento Desenvolv Hum 2007; 17(2):01-11.
\end{abstract}

Resumo: Há controvérsias quanto ao crescimento de crianças em aleitamento exclusivo (AME) dos três aos seis meses de idade, embora a Organização Mundial de Saúde afirme que o AME até os seis meses de vida garante um crescimento adequado. $\mathrm{O}$ objetivo deste estudo foi medir a velocidade média de incremento diário de peso (VIDP) no dois primeiros trimestres de vida, segundo a duração do aleitamento exclusivo (AME), ajustada segundo outros possíveis determinantes do crescimento. Foram estudadas 347 crianças participantes de um estudo de coorte de recém nascidos, nos anos de 1998 e 1999, em um hospital universitário de São Paulo. Análises de regressão linear múltipla foram realizadas para investigar o efeito da duração do AME na VIDP, utilizando-se modelo hierarquizado. Resultados: 17,5\% das crianças estavam em AME aos três meses; $2,4 \%$ aos seis meses. No primeiro trimestre, entre as meninas, maiores VIDP estão relacionadas a maior duração do AME; entre os meninos, a VIDP não difere segundo duração do AME. A VIDP de crianças no segundo trimestre não é influenciada pela duração do AME. Conclui que o AME garante crescimento adequado nos dois primeiros trimestres de vida; as aparentes alterações no crescimento de crianças em AME devem ser avaliadas com cautela, a fim de evitar a introdução desnecessária de alimentação complementar.

Palavras-chave: Crescimento. Nutrição infantil. Aleitamento materno. Regressão linear.

\section{INTRODUÇÃO}

O crescimento no primeiro ano de vida é um processo complexo com mudanças rápidas, sendo afetado direta ou indiretamente por diversos fatores, como sexo, raça, o peso ao nascer, condições de saúde da criança e tipo de alimentação. Esses fatores são influenciados por condições socioeconômicas, culturais e biológicas ${ }^{15,18}$. Portanto, diante de sua natureza multicausal, o crescimento infantil deve ser avaliado levando em consideração fatores relacionados com a sua etiologia e ao seu desenvolvimento ${ }^{15}$. $\mathrm{A} \mathrm{OMS}^{25}$ recomenda que durante os seis primeiros meses de vida os lactentes devam ser alimentados exclusivamente com leite materno, pois nessa fase ele é o único alimento ideal para o crescimento e desenvolvimento da criança.

Apesar das inúmeras evidências da importância do aleitamento materno exclusivo nos

\footnotetext{
Departamento de Epidemiologia da Faculdade de Saúde Pública- Universidade de São Paulo. São Paulo, SP. Brasil. rosanut@usp.br Doutoranda em Nutrição Humana Aplicada - PRONUT - FCF/FEA/FSP- Universidade de São Paulo.

** Prof. Dr. Titular do Departamento de Epidemiologia da Faculdade de Saúde Pública-USP.

Este artigo foi baseado na dissertação de mestrado da primeira autora intitulado "Crescimento de crianças no $1^{\circ}$ e no $2^{\circ}$ trimestres de vida, segundo duração do aleitamento materno exclusivo" apresentado no Programa de Pós-Graduação Interunidades em Nutrição Humana Aplicada- PRONUT da FCF/FEA/FSP da Universidade de São Paulo no ano de 2005.
} 
primeiros seis meses, há controvérsias sobre o padrão de crescimento relacionado ao tipo de aleitamento neste período ${ }^{5,23}$. É consenso que o aleitamento materno exclusivo (AME) proporciona um crescimento ótimo de zero aos três meses de idade. Já dos três aos seis meses de vida, alguns estudos em países em desenvolvimento mostram que nesse período a curva ponderal de crianças alimentadas exclusivamente ao seio desvia-se um pouco para baixo em relação às curvas de referência disponíveis. A interpretação desse fenômeno não é unânime: para alguns ${ }^{22}$, representa uma insuficiência de crescimento; para outros, é normal, e as curvas de referência é que não são adequadas ${ }^{4,12,18}$.

As curvas do National Center for Health Statistics (NCHS, 1977) foram, até recentemente, o padrão de referência recomendado para uso internacional pela OMS e adotado pelo Ministério da Saúde do Brasil, permitindo comparações de dados entre diferentes populações, no entanto, essas curvas basearam-se, predominantemente, nas medidas de crianças brancas, de classe média, alimentadas artificialmente e com introdução precoce de alimentos sólidos, devendo, por isso, ter seu uso avaliado com cautela9.

A interpretação da trajetória de crescimento dos bebês alimentados com leite materno, muitas vezes utilizada para avaliar até que ponto a alimentação do bebê está adequada, permitindo atingir o potencial de crescimento, assim como para orientar a respeito da introdução de alimentos complementares, é altamente dependente dos dados de referência utilizados. Assim, a interpretação do crescimento infantil pode ser prejudicada se o padrão utilizado como referência não representar adequadamente o padrão de crescimento fisiológico de bebês saudáveis alimentados com leite materno.

Em abril de 2006, a OMS divulgou uma nova referência internacional de crescimento infantil, que se baseia em crianças alimentadas até 6 meses, predominantemente com leite materno ${ }^{27}$.

Outra forma de se avaliar o crescimento é calculando sua velocidade. Ao acompanhar o crescimento de uma criança, o parâmetro mais importante a ser considerado é a velocidade de crescimento, pois essa apontará de forma mais criteriosa para o problema de crescimento ${ }^{26}$.
Um aspecto importante a ser considerado em estudos que relacionam crescimento com o tipo de alimentação infantil é como classificar a criança quanto à sua alimentação, já que mudanças podem ocorrer durante o período avaliado. Estudos de seguimento de crianças ao nascer, com observação diária da alimentação, permitem identificar com maior validade a idade de introdução de alimentos, possibilitando calcular o tempo de permanência em AME, o que confere melhor interpretação de sua influência no crescimento infantil.

O presente estudo objetivou medir o efeito da duração do AME na velocidade de incremento médio diário de peso, nos dois primeiros trimestres de vida, ajustado com outros possíveis fatores do processo de crescimento.

\section{MÉTODOS}

Os dados utilizados são provenientes de uma pesquisa matriz desenvolvida no Departamento de Epidemiologia da Faculdade de Saúde Pública da Universidade de São Paulo, que teve por objetivo estudar a alimentação e o crescimento no primeiro ano de vida. Trata-se de um estudo prospectivo de coorte de crianças nascidas em um hospital universitário, situado no município de São Paulo.

A seleção das mães participantes, internadas na ocasião do parto na maternidade, começou em outubro de 1998 e terminou em novembro de 1999, mantendo um ritmo médio de recrutamento de quatro mães por dia. Não foram incluídas no estudo crianças nascidas de parto gemelar, crianças com peso ao nascer inferior a $2.500 \mathrm{~g}$ ou que apresentaram intercorrências.

A dinâmica da coleta de dados ocorreu por meio de consultas ao prontuário das mães no dia do parto, entrevistas por meio de questionários estruturados, no hospital e na residência e, ainda, anotações diárias maternas sobre a alimentação e morbidade da criança. Foram feitas sete visitas domiciliares programadas para 15,30, 60, 90, 180, 270 e 360 dias de idade da criança; nessas visitas, entrevistadoras treinadas realizavam medições antropométricas e aplicavam o questionário. 
O tamanho inicial da coorte matriz foi 506 crianças, com 20,9\% de perda, totalizando 347 crianças com informações completas de alimentação e das medidas peso até os seis meses de idade. O principal motivo dessas perdas foi mudança de endereço $(83,7 \%)$.

Foi realizado estudo ${ }^{+}$para análise das perdas e não se observaram diferenças estatisticamente significantes entre as características das crianças que completaram o seguimento e as que deixaram de participar.

Para o presente estudo utilizaram-se os dados colhidos na maternidade, na quarta visita domiciliar (em torno de 90 dias) e quinta visita domiciliar (em torno de 180 dias).

A variável explanatória principal "duração do AME no período" foi categorizada em três intervalos de tempo para cada trimestre. Optouse pela categorização porque a distribuição das crianças, segundo a duração do AME, apresentou comportamento muito irregular no decorrer do acompanhamento. Observou-se alta concentração de crianças que deixaram o AME no primeiro trimestre, diminuindo, assim, o tamanho amostral no segundo trimestre, principalmente, no final do período.

O processo de introdução de alimentos não ocorreu de maneira regular e contínua; muitas vezes, o AME era interrompido (com introdução de outros alimentos) e, logo após um período curto, retomado, permanecendo até o final do período estudado. Diante disso, julgouse que não seria apropriado considerar como interrompido o AME a partir, simplesmente, da primeira oferta de algum outro alimento. Assim, considerou-se que as crianças estudadas deixam o aleitamento materno exclusivo quando outros alimentos (líquido ou sólido) são oferecidos com uma freqüência de pelo menos três vezes, em um período de sete dias, repetindo durante pelo menos um mês.

As crianças foram pesadas e medidas na quarta e quinta visita (segundo trimestre), sem ocorrer precisamente aos 90 e aos 180 dias de idade. Em razão dessa variação, a duração do AME no primeiro trimestre e no segundo trimestre foi descrita em percentagem de tempo, por meio da seguinte fórmula:

Duração do AME $(\%)=\left(\begin{array}{c}\text { Duração (em dias) do } \\ \text { AME no período } \\ \begin{array}{c}\text { Tempo de observação no } \\ \text { período (em dias) }\end{array}\end{array}\right) \times 100$

O crescimento (variável resposta) foi medido por meio da velocidade de incremento médio diário de peso (VIDP) em cada período:

$$
\operatorname{VIDP}(\mathrm{g} / \text { dia })=\frac{\text { peso final }(\mathrm{g})-\text { peso inicial }(\mathrm{g})}{\text { tempo de observação }(\text { em dias })}
$$

O tempo de observação utilizado nos cálculos corresponde à diferença entre a idade no final do período estudado e a idade no início do período.

Foram realizadas análises de regressão linear múltipla para investigar o efeito da duração do AME na velocidade de ganho de peso, controlando o efeito das outras variáveis de interesse selecionadas, com modelo hierarquizado teórico ${ }^{21}$ esquematizado na Figura 1.

A estrutura da hierarquização foi elaborada considerando um marco teórico com as variáveis associadas com o crescimento infantil. O marco teórico priorizou o efeito do aleitamento materno exclusivo, adotando-o como determinante proximal do crescimento e ajustando seu efeito segundo as demais características estudadas.

$\mathrm{Na}$ modelagem, as variáveis contínuas (idade da mãe, peso ao nascer e peso e no início do segundo trimestre) foram transformadas em variáveis centralizadas, tendo a média como posição de referência (0). Todas as demais variáveis independentes, de possível confusão ou interação, foram transformadas em variáveis indicadoras, assumindo o valor 0 ou 1 . Na ausência de informação para alguma variável, o dado foi registrado como ausente e o indivíduo retirado da análise.

\footnotetext{
Augusto RAA, Souza JMP. Estudo comparativo das características de mães participantes e desistentes de um estudo de coorte contemporânea intitulado Alimentação no Primeiro ano de vida”. In: Anais do VII Congresso Brasileiro de Saúde Coletiva. Livro de Resumos II. Brasília, Brasil. Brasília: Associação Brasileira de Pós-graduação em Saúde Coletiva- ABRASCO; 2003. p. 321.
} 
Figura 1: Modelo teórico de investigação dos efeitos de fatores relacionados ao crescimento infantil, durante os seis primeiros meses de vida, estruturado em blocos hierarquizados

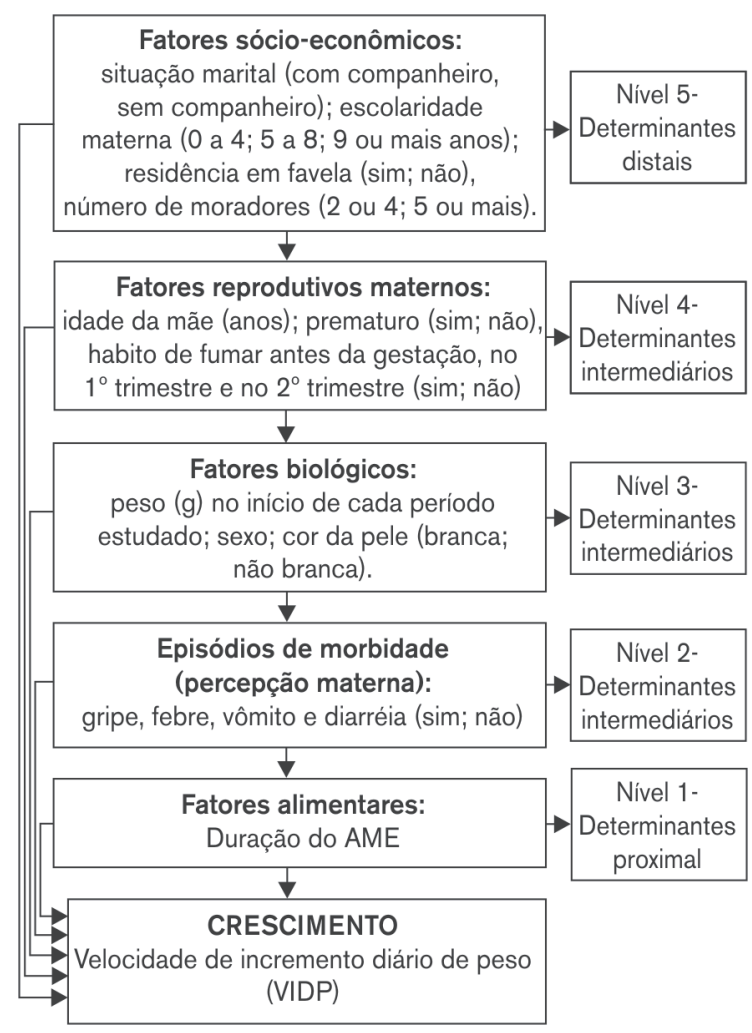

As variáveis independentes não binárias estão descritas no Quadro 1 e as variáveis categóricas binárias no Quadro 2.

Quadro 1: Valores assumidos pelas variáveis independentes não binárias.

\begin{tabular}{|c|c|c|c|}
\hline \multirow{2}{*}{$\begin{array}{l}\text { Variável } \\
\text { independente }\end{array}$} & \multirow{2}{*}{\begin{tabular}{|l|} 
Variável \\
indicadora
\end{tabular}} & \multicolumn{2}{|c|}{ Valor } \\
\hline & & 1 & 0 \\
\hline \multirow{3}{*}{$\begin{array}{l}\text { Duração do } \\
\text { aleitamento } \\
\text { materno } \\
\text { exclusivo no } \\
1^{\circ} \text { trimestre } \\
\text { (ame } 1) \text {. }\end{array}$} & $\begin{array}{l}\text { ame } 1 \mathrm{~T} \_1 \\
\text { (referência) }\end{array}$ & 0 a 29 dias. & Se diferente \\
\hline & ame 1T_2 & 30 a 59 dias. & Se diferente \\
\hline & ame 1T_3 & 60 ou mais dias. & Se diferente \\
\hline \multirow{3}{*}{$\begin{array}{l}\text { Duração do } \\
\text { aleitamento } \\
\text { materno } \\
\text { exclusivo no } \\
2^{\circ} \text { trimestre } \\
(\text { ame2). }\end{array}$} & $\begin{array}{l}\text { ame 2T_1 } \\
\text { (referência) }\end{array}$ & Ausência de ame2 & Se diferente \\
\hline & ame 2T_2 & 1 a 29 dias. & Se diferente \\
\hline & ame 2T_3 & 30 ou mais dias. & Se diferente \\
\hline \multirow{3}{*}{$\begin{array}{l}\text { Escolaridade } \\
\text { materna } \\
\text { (anos de } \\
\text { estudo) }\end{array}$} & \begin{tabular}{|l} 
esc 1 \\
(referência)
\end{tabular} & $\begin{array}{l}0 \text { a } 4 \text { anos de } \\
\text { estudo. }\end{array}$ & Se diferente \\
\hline & esc 2 & $\begin{array}{l}5 \text { a } 8 \text { anos de } \\
\text { estudo. }\end{array}$ & Se diferente \\
\hline & esc 3 & $\begin{array}{l}9 \text { ou mais anos de } \\
\text { estudo. }\end{array}$ & Se diferente \\
\hline
\end{tabular}

Quadro 2 - Valores assumidos pelas variáveis categóricas binárias.

\begin{tabular}{|c|c|c|}
\hline \multirow[t]{2}{*}{ Variável } & \multicolumn{2}{|c|}{ Valor } \\
\hline & 0 & 1 \\
\hline Situação marital & $\begin{array}{l}\text { Sem } \\
\text { companheiro }\end{array}$ & $\begin{array}{l}\text { Com } \\
\text { companheiro }\end{array}$ \\
\hline Residência em favela & Morador & Não moradol \\
\hline Prematuro & \begin{tabular}{|l|}
$\operatorname{Sim}(<37$ \\
semanas de \\
gestação)
\end{tabular} & $\begin{array}{l}\text { Não (>=37 } \\
\text { semanas de } \\
\text { gestação) }\end{array}$ \\
\hline $\begin{array}{l}\text { Hábito de fumar da mãe } \\
\text { antes da gestação }\end{array}$ & Fumante & Não fumante \\
\hline $\begin{array}{l}\text { Hábito de fumar da mãe } \\
\text { durante o } 1^{\circ} \text { trimestre }\end{array}$ & Fumante & Não fumante \\
\hline $\begin{array}{l}\text { Hábito de fumar da mãe } \\
\text { durante o } 2^{\circ} \text { trimestre }\end{array}$ & Fumante & Não fumante \\
\hline Número de moradores & 2 a 4 & 5 ou mais \\
\hline Cor da pele da criança & Não branca & Branca \\
\hline Sexo da criança & Feminino & Masculino \\
\hline
\end{tabular}

Foram medidos os efeitos da duração do aleitamento materno exclusivo em comparação com a categoria de referência (menor duração do aleitamento exclusivo no período). Quando necessário, realizou-se teste de combinação linear entre duas categorias. A equação de regressão completa para cada trimestre foi:

$\overline{\text { VIDP }}=\boldsymbol{\beta}_{0}+\boldsymbol{\beta}_{1}$ marital $+\boldsymbol{\beta}_{2}$ escolaridade materna $2+\boldsymbol{\beta}_{3}$ escolaridade materna $3+\boldsymbol{\beta}_{4}$ favela $\boldsymbol{+} \boldsymbol{\beta}_{5}$ numero moradores $+\boldsymbol{\beta}_{6}$ fumo antes gestação $+\boldsymbol{\beta}_{7}$ fumo no trimestre $+\boldsymbol{\beta}_{8}$ prematuro $+\boldsymbol{\beta}_{11}$ idade mãe $+\boldsymbol{\beta}_{12}$ peso no início do período $+\boldsymbol{\beta}_{13}$ sexo $+\boldsymbol{\beta}_{14}$ cor $+\boldsymbol{\beta}_{15}$ febre $+\boldsymbol{\beta}_{16}$ gripe $+\boldsymbol{\beta}_{17}$ vômito + $\boldsymbol{\beta}_{18}$ diarréia+ $\boldsymbol{\beta}_{19}$ segundo intervalo do AME no período + $\boldsymbol{\beta}_{20}$ terceiro intervalo do AME no período.

Os coeficientes de regressão foram calculados com os respectivos intervalos com $95 \%$ de confiança, com apresentação dos níveis descritivos $\mathrm{P}$ dos testes.

A modelagem partiu de análises simples, entre a variável resposta e cada uma das variáveis estudadas; entraram no modelo as variáveis com $p \leq 0,25$. As variáveis selecionadas foram incluídas na análise múltipla em seus respectivos níveis hierárquicos, continuando na modelagem as variáveis com $p \leq 0,10$. A análise múltipla seguiu agregando-se as variáveis selecionadas em cada nível hierárquico, iniciando com as variáveis que permaneceram no quarto e quinto níveis. $\mathrm{Na}$ agregação dos níveis, as variáveis selecionadas 
do quinto nível permaneceram na análise subsequiente, independentemente do valor de $\mathrm{p}$. Para as variáveis dos níveis inferiores, o critério de permanência foi $\mathrm{p} £ 0,10$.

A modelagem continuou seguindo o mesmo procedimento para os demais níveis. As variáveis relacionadas à alimentação, à morbidade, o peso e o sexo permaneceram no modelo independentemente do valor de $\mathrm{p}$.

As análises foram feitas em separado para cada período estudado. Para o processamento dos dados utilizou-se o programa Stata $^{20}$ versão 8.0.

A pesquisa foi aprovada pelo Comitê de Ética da Faculdade de Saúde Pública, segundo a resolução no 196 de 10 de outubro de 1996 do Conselho Nacional de Saúde.

\section{RESULTADOS}

A amostra tem distribuição uniforme segundo sexo; consiste em sua maioria de crianças de cor branca, nascidas de parto normal, não prematuras, não moradoras de favela e filhas de mães não fumantes. Do total de mães, $45 \%$ possuíam mais de nove anos de estudo, mais da metade das crianças morava com menos de três pessoas na casa e, ainda, $81 \%$ das crianças contavam com a presença do pai no domicílio (Tabela 1). A mediana da idade materna foi 25 anos .

A mediana do peso e do comprimento ao nascer foi $3.250 \mathrm{~g}$ e $49 \mathrm{~cm}$, respectivamente. Avaliando a evolução do peso, notam-se aumentos médios de $84 \%$ no primeiro trimestre e $28 \%$ no segundo trimestre.

Quanto à ocorrência de episódios de morbidade no primeiro trimestre, observou-se maior prevalência de gripe $(66,6 \%)$ seguida por febre $(28,8 \%)$, e vômito $(23,1 \%)$ e, em menor proporção $(6,9 \%)$, aparece diarréia (Tabela 2$)$. No segundo trimestre, a ordem de agravos é a mesma, com aumento na prevalência dos episódios de gripe, febre e diarréia $(72,9 \% ; 37,2 \%$ e $9,8 \%$ respectivamente), exceto para vômito $(21,3 \%)$ (Tabela 2).
Tabela 1 - Distribuição das crianças, segundo variáveis categóricas. São Paulo, 1999.

\begin{tabular}{|c|c|c|}
\hline Variáveis & $\mathbf{n}$ & $\%$ \\
\hline \multicolumn{3}{|l|}{ Sexo } \\
\hline Masculino & 176 & 50,7 \\
\hline Feminino & 171 & 49,3 \\
\hline \multicolumn{3}{|l|}{ Cor } \\
\hline Branca & 141 & 59,4 \\
\hline Não Branca & 206 & 40,6 \\
\hline \multicolumn{3}{|l|}{ Prematuro } \\
\hline Sim & 12 & 3,5 \\
\hline Não & 329 & 94,8 \\
\hline sem informação & 6 & 1,7 \\
\hline \multicolumn{3}{|l|}{ Residência em favela } \\
\hline Sim & 122 & 35,2 \\
\hline Não & 225 & 64,8 \\
\hline \multicolumn{3}{|l|}{$\begin{array}{l}\text { Hábito de fumar da mãe } \\
\text { antes da gestação }\end{array}$} \\
\hline Fumante & 76 & 21,9 \\
\hline Não fumante & 271 & 78,1 \\
\hline \multicolumn{3}{|l|}{$\begin{array}{l}\text { Hábito de fumar da mãe } \\
\text { durante o } 1^{\circ} \text { trimestre }\end{array}$} \\
\hline Fumante & 58 & 16,7 \\
\hline Não fumante & 289 & 83,3 \\
\hline \multicolumn{3}{|l|}{$\begin{array}{l}\text { Hábito de fumar da mãe } \\
\text { durante o } 2^{\circ} \text { trimestre }\end{array}$} \\
\hline Fumante & 58 & 16,7 \\
\hline Não fumante & 289 & 83,3 \\
\hline \multicolumn{3}{|l|}{ Escolaridade materna } \\
\hline 0 a 4 anos & 58 & 16,7 \\
\hline 5 a 8 anos & 133 & 38,3 \\
\hline 9 ou mais anos & 156 & 45,0 \\
\hline \multicolumn{3}{|l|}{ Número de moradores } \\
\hline 2 a 4 & 179 & 51,9 \\
\hline 5 ou mais & 166 & 48,1 \\
\hline \multicolumn{3}{|l|}{ Condição marital da mãe } \\
\hline Sem companheiro & 66 & 19,0 \\
\hline Com companheiro & 281 & 81,0 \\
\hline \multicolumn{3}{|l|}{$\begin{array}{l}\text { Duração do AME no } 1^{\circ} \\
\text { trimestre }\end{array}$} \\
\hline $0-30$ & 172 & 49,57 \\
\hline $30-60$ & 68 & 19,60 \\
\hline 60 - ou mais & 107 & 30,84 \\
\hline \multicolumn{3}{|l|}{$\begin{array}{l}\text { Duração do AME no } 2^{\circ} \\
\text { trimestre }\end{array}$} \\
\hline $0 *$ & 286 & 82,42 \\
\hline $0-30$ & 21 & 6,05 \\
\hline $30-60$ & 19 & 5,48 \\
\hline 60 - ou mais & 21 & 6,05 \\
\hline
\end{tabular}

* Crianças que desmamaram no $1^{\circ}$ trimestre 
Tabela 2 - Distribuição das crianças, segundo episódios de morbidade. São Paulo, 1999.

\begin{tabular}{|l|r|r|r|r|}
\hline \multirow{2}{*}{ Morbidade } & \multicolumn{2}{|c|}{$\mathbf{1}^{\mathbf{0}}$ Trimestre } & \multicolumn{2}{c|}{$\mathbf{2}^{\mathbf{0}}$ Trimestre } \\
\cline { 2 - 5 } & $\mathbf{n}$ & $\mathbf{\%}$ & $\mathbf{n}$ & \% \\
\hline Febre & 100 & 28,8 & 129 & 37,2 \\
\hline Sim & 247 & 71,2 & 218 & 62,8 \\
\hline Não & 231 & 66,6 & 253 & 72,9 \\
\hline Gripe \\
\hline Sim & 116 & 33,4 & 94 & 27,1 \\
\hline Não & 24 & 6,9 & 74 & 9,8 \\
\hline Diarréia & 323 & 93,1 & 313 & 90,2 \\
\hline Sim & 80 & 23,1 & 74 & 21,3 \\
\hline Não & 267 & 76,9 & 273 & 78,7 \\
\hline Vômito &
\end{tabular}

O Gráfico 1 apresenta a percentagem de crianças que permaneceram em AME no decorrer dos primeiros seis meses de vida. Metade das crianças deixou o AME durante o primeiro mês de vida e menos de $18 \%$ continuaram por um período superior a 90 dias. No segundo trimestre, a maioria das crianças $(82,4 \%)$ iniciou o período sem AME e apenas 2,0\% encontravam-se em AME aos 180 dias.

Gráfico 1 - Percentagem de crianças que permanecem em aleitamento materno exclusivo ao longo do $1^{\circ}$ semestre. São Paulo, 1999.

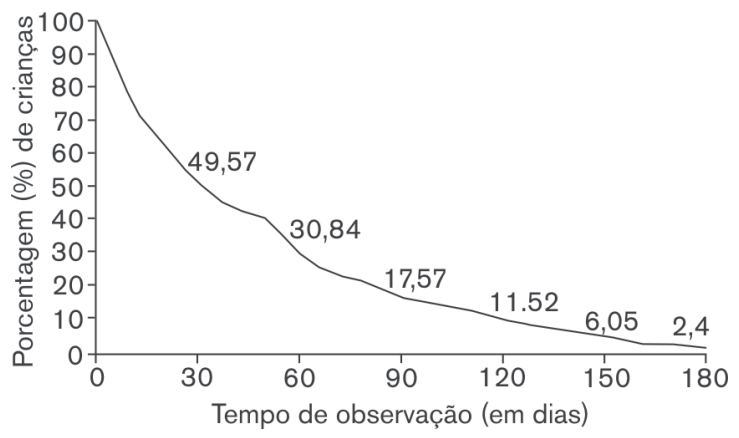

De acordo com as médias da VIDP não ajustadas (modelo bruto), no segundo trimestre a velocidade diminui, sendo em média $10 \mathrm{~g} / \mathrm{dia}$ menor que no primeiro trimestre: $28,8 \mathrm{~g} / \mathrm{dia}-$ 18,4g/dia (Tabela 3).
Adotando-se nível de significância a = 5\%, os dados do modelo hierarquizado ajustado final (Tabela 3), indicam significância para as variáveis: idade da mãe, peso ao nascer, sexo, duração do AME e para a interação sexo vs. AME. Essa interação foi explorada, estratificando-se o efeito do AME na VIDP1 (primeiro trimestre), segundo sexo. No sexo feminino, as médias de VIDP1 das crianças pertencentes à categoria de maior duração do AME apresentam-se significativamente maiores que das categorias de menor duração. No sexo masculino, não foi observada diferença. De maneira geral, no primeiro trimestre, maiores VIDP estão relacionadas a maior idade materna, a menor peso ao nascer e ao sexo masculino. Independentemente da categoria de AME, as VIDP1 são maiores para o sexo masculino quando comparadas ao sexo feminino, sendo $32,07 \mathrm{~g} /$ dia e $26,8 \mathrm{~g}$ /dia respectivamente.

No modelo ajustado final para VIDP2 (segundo trimestre), as características maior idade materna, mãe não fumante e menor peso no início do período estão relacionados com menores VIDP2. A duração do aleitamento exclusivo parece não influenciar a VIDP2 (Tabela 3 ).

A diferença encontrada entre a VIDP2 de crianças filhas de mães fumantes e de mães não fumantes foi melhor explorada por meio de comparações entre suas características. Verificouse que filhos de mães fumantes apresentam peso de nascimento significativamente menor (3.286 $\pm 390 \mathrm{~g}$ ) do que filhos de mães não fumantes $(3.152 \pm 367 \mathrm{~g})$.

\section{DISCUSSÃO}

Decidiu-se não trabalhar com a variável AME em sua forma contínua, devido ao grande abandono do AME ao longo dos primeiros seis meses de vida, principalmente no $1^{\circ}$ trimestre, o que acarretou em grande redução da amostra de crianças em AME. Esse comportamento acompanha a realidade nacional. Estudos de base populacional $^{2,7}$ revelam baixas prevalências de AME aos seis meses de vida e destacam que o abandono dessa prática ocorre de forma mais acentuada logo nos primeiros três meses de vida. 
Tabela 3: Coeficientes de regressão linear múltipla, respectivos intervalos de confiança e níveis descritivos do teste para velocidade de

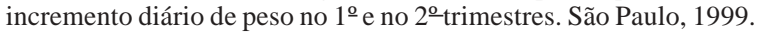

\begin{tabular}{|c|c|c|c|c|c|c|c|}
\hline \multirow[t]{2}{*}{ Variáveis e Modelos } & \multicolumn{3}{|c|}{ VIDP $1^{\circ}$ trimestre (VIDP1) } & \multirow[t]{2}{*}{ Variáveis e Modelos } & \multicolumn{3}{|c|}{ VIDP $2^{\circ}$ trimestre (VIDP2) } \\
\hline & Coef. & (IC95\%) & & & Coef. & (IC95\%) & \\
\hline Modelo Bruto & & & & Modelo Bruto & & & \\
\hline Interceptação $\left(\beta_{0}\right)$ & 28,58 & $(27,59-29,60)$ & 0,000 & Interceptação $\left(\beta_{0}\right)$ & 18,36 & $(17,71-19,01)$ & 0,000 \\
\hline Duração do AME & & & & Duração do AME & & & \\
\hline $0-30{\text { (refer })^{(\mathrm{a})}}$ & 0 & $(-1,60-2,11)$ & 0,785 & 0 dia (refer) & 0 & - & - \\
\hline $30-60^{(\mathrm{b})}$ & 0,26 & $(1,05-4,24)$ & 0,001 & $0-30$ & $-0,88$ & $(-3,37-1,61)$ & 0,488 \\
\hline $260^{(\mathrm{c})}$ & 2,64 & $(0,38-4,39)$ & 0,020 & $\geq 30$ & $-1,05$ & $(-2,91-0,81)$ & 0,268 \\
\hline Comparação $(c-b)$ & 2,38 & & & & & & \\
\hline Modelo Ajustado & & & & Modelo Bruto & & & \\
\hline Interceptação $\left(\beta_{0}\right)$ & 25,91 & $(22,76-29,05)$ & 0,000 & Interceptação $\left(\beta_{0}\right)$ & 20,17 & $(17,28-23,05)$ & 0,000 \\
\hline Duração do AME & & & & Duração do AME & & & \\
\hline $0-30{\text { (refer })^{(\mathrm{a})}}$ & 0 & $(-1,86-2,57)$ & 0,978 & 0 dia (refer) & 0 & $(-3,44-1,53)$ & 0,451 \\
\hline $30-60^{(b)}$ & 0,35 & $(0,97-5,63)$ & 0,006 & $0-30$ & $-0,95$ & $(-3,12-0,67)$ & 0,205 \\
\hline $260^{(\mathrm{c})}$ & 3,30 & $(0,25-5,65)$ & 0,033 & 230 & $-1,22$ & & \\
\hline Comparação $(c-b)$ & 2,95 & & & & & & \\
\hline Escolaridade materna & & & & Escolaridade materna & & & \\
\hline $0-5 \operatorname{anos}(\mathrm{ref})^{(\mathrm{a})}$ & & & & $0-5 \operatorname{anos}(\mathrm{ref})^{(\mathrm{a})}$ & 0 & & \\
\hline $5-9$ anos (b) & & & & $5-9 \operatorname{anos}{ }^{(b)}$ & 0,933 & $(-0,82-2,68)$ & 0,295 \\
\hline $9 \mid$ - ou mais ${ }^{(c)}$ & - & - & & $9 \mid$ - ou mais ${ }^{(c)}$ & 0,460 & $(-1,23-2,15)$ & 0,592 \\
\hline $\operatorname{Lincon}(c-b)$ & & & & $\operatorname{Lincon}(c-b)$ & & & \\
\hline Situação Marital & & & & Situação Marital & & & \\
\hline Sem companheiro & 0 & & & Sem companheiro & & & \\
\hline Com companheiro & 1,05 & $(-2,731-0,63)$ & & Com companheiro & - & - & - \\
\hline Idade mãe (em anos) & 0,13 & $(0,02-0,24)$ & 0,020 & Idade mãe (em anos) & 0,115 & $(-0,22--0,02)$ & 0,024 \\
\hline Hábito de fumar & & & & Hábito de fumar & & & \\
\hline Sim & - & - & - & Sim & 0 & & \\
\hline Não & - & - & - & Não & $-2,313$ & $(-3,92--0,71)$ & 0,005 \\
\hline Peso ao Nascer (g) & 0,002 & $(-0,004--0,00)$ & 0,013 & Peso Início do $2^{\circ} \mathrm{T}$ (g) & 0,001 & $(0,000-0,002)$ & 0,050 \\
\hline Sexo & & & & Sexo & & & \\
\hline Feminino (ref) & 0 & & & Feminino (ref) & 0 & & \\
\hline Masculino & 6,20 & $(4,40-8,00)$ & 0,000 & Masculino & 0,407 & $(-089-1,70)$ & 0,537 \\
\hline Gripe & & & & Gripe & & & \\
\hline Sim & 0 & & & Sim & 0 & & \\
\hline Não & 0,72 & $(-2,14-0,68)$ & 0,309 & Não & 0,862 & $(-0,59-2,31)$ & 0,243 \\
\hline Febre & & & & Febre & & & \\
\hline Sim & 0 & & & Sim & 0 & & \\
\hline Não & $-1,44$ & $(-2,88-0,003)$ & 0,062 & Não & $-1,031$ & $(-2,37-0,31)$ & 0,131 \\
\hline Diarréia & & & & Diarréia & & & \\
\hline Sim & 0 & & & Sim & 0 & & \\
\hline Não & 0,72 & $(-0,58-4,50)$ & 0,130 & Não & $-1,247$ & $(-3,30-0,81)$ & 0,233 \\
\hline Vômito & & & & Vômito & & & \\
\hline Sim & 0 & & & Sim & 0 & & \\
\hline Não & 0,35 & $(-1,20-1,90)$ & 0,660 & Não & 1,161 & $(-0,36-2,69)$ & 0,135 \\
\hline
\end{tabular}




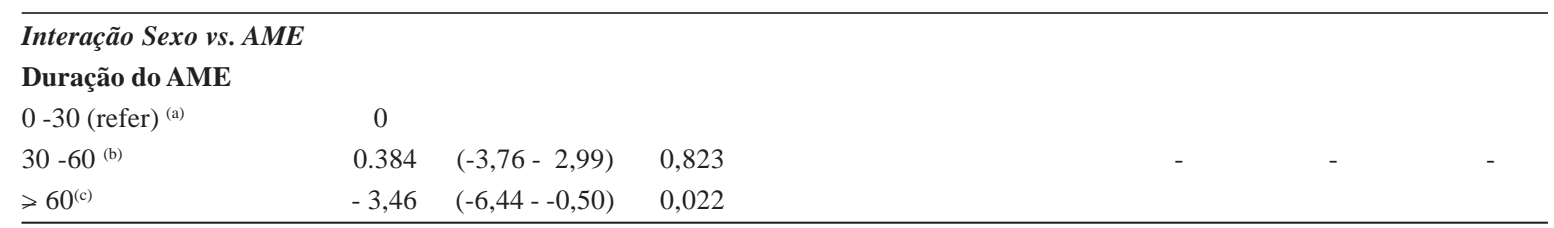

\begin{tabular}{|c|c|c|c|c|c|c|}
\hline \multicolumn{7}{|c|}{ Estratificação segundo sexo } \\
\hline \multicolumn{7}{|l|}{ Feminino } \\
\hline $0-30$ (refer) $^{\text {(a) }}$ & 0 & & & & & \\
\hline $30-60^{(b)}$ & 0,032 & $(-2,15-2,22)$ & 0,977 & & & \\
\hline $260^{(\mathrm{c})}$ & 3,372 & $(1,08-5,66)$ & 0,004 & - & - & - \\
\hline Comparação (c - b) & 3,340 & $(0,67-6,01)$ & 0,014 & & & \\
\hline \multicolumn{7}{|l|}{ Masculino } \\
\hline $0-30$ (refer) $^{(a)}$ & 0 & & & & & \\
\hline $30-60$ (b) & $-0,352$ & $(-2,95-2,25)$ & 0,790 & & & \\
\hline $260^{(\mathrm{c})}$ & $-0,095$ & $(-2,02-1,83)$ & 0,923 & - & - & - \\
\hline Comparação (c - b) & 0,257 & $(-2,38-2,89)$ & 0,848 & & & \\
\hline
\end{tabular}

Quanto aos eventos de morbidade segundo a percepção materna, as maiores ocorrências são observadas no segundo trimestre, período em que se observa, também, um menor número de crianças em AME. Alguns trabalhos ${ }^{10,17}$ têm mostrado que a maior propensão para as infecções respiratórias e episódios de diarréia acontece quando o AME é substituído por alimentos com baixos valores nutricionais, e sujeitos a contaminação. No entanto, não foram verificadas associações entre a ocorrência de episódios de morbidade e a velocidade de incremento diário de peso, tanto no primeiro quanto no segundo trimestre.

Ao comparar os resultados obtidos com os de outros estudos, pode-se admitir que crianças pertencentes à categoria de maior duração do AME são as representantes mais próximas de crianças que permanecem todo o período em AME e que as crianças das demais categorias (menores durações do AME) representam crianças em outros tipos de alimentação.

$\mathrm{O}$ crescimento de crianças em função do AME apresenta comportamento diferente segundo sexo no primeiro trimestre. Para o sexo feminino, a maior duração do AME está associada a maiores valores de VIDP1; no sexo masculino, a VIDP1 não é explicada pela duração do AME. Comportamento semelhante ao observado para o sexo feminino foi descrito por Longo et $\mathrm{al}^{8}$.

Além do sexo e do AME, a VIDP1 também sofre influência de outros fatores, entre eles, a idade materna e o peso ao nascer. A cada um ano de aumento na idade da mãe, é verificado um incremento de 0,138g/dia na VIDP1. A influência da maior idade materna na promoção de maior crescimento também foi encontrado por Puccini et al. ${ }^{16}$. Em teoria, uma possível explicação seria a maior inexperiência da mãe mais jovem nos aspectos relacionados à alimentação e aos cuidados gerais com a criança.

Quanto ao peso ao nascer, crianças nascidas com menor peso apresentam maior VIDP1, isso provavelmente ocorra na tentativa do organismo em recuperar alguma limitação sofrida em seu potencial. Esse comportamento também foi encontrado por Longo et al. ${ }^{8}$.

Diferentemente do primeiro trimestre, a velocidade de incremento diário de peso no $2^{\circ}$ trimestre (VIDP2) não é influenciada pela duração do aleitamento exclusivo, indicando que o crescimento de crianças em AME é semelhante ao de crianças em outras formas de alimentação, concordando com alguns estudos ${ }^{5,3,11}$. Porém, discordam de outros ${ }^{12,24}$, que encontraram menor crescimento no segundo trimestre em crianças em AME quando comparado ao crescimento de crianças em outras formas de alimentação. No entanto, é importante considerar que esses estudos basearam-se em avaliações pontuais, utilizando outro grupo populacional como comparação (crianças do NCHS) e não consideraram o efeito de outros fatores relacionados ao crescimento.

Outras variáveis apresentaram-se relacionadas à VIDP2, como idade materna, peso 
no início e hábito de fumar materno. À medida que aumenta o peso no início do período, a VIDP2 também aumenta. Maior idade materna, diferentemente do primeiro trimestre, levam ao menor incremento de peso, não sendo encontrada na literatura nenhuma explicação para esse comportamento.

Em relação ao hábito de fumar materno, os resultados mostraram que filhos de mães não fumantes apresentam menor velocidade de incremento de peso. Devido a todos os efeitos negativos do hábito de fumar materno na saúde da mãe e da criança, esses resultados foram explorados, na tentativa de se obter alguma explicação para tal ocorrência. Assim, foram realizadas comparações entre a variável "hábito de fumar materno" e as variáveis que apresentaram efeito significante na VIDP tanto no segundo como no primeiro trimestre. Verificou-se que filhos de mães fumantes apresentaram peso ao nascer significativamente menor do que filhos de mães não fumantes. Siqueira et al., ${ }^{19}$ estudando hábito de fumar materno e crescimento pós-natal, observaram que, após o quarto mês de vida, filhos de mães fumantes apresentaram maior ganho de peso e altura em relação às não fumantes, o que os autores justificam como uma possível retomada compensatória do crescimento, devido a agravo provocado em período anterior. O resultado encontrado no presente estudo também pode ser justificado com a mesma explicação, uma vez que filhos de mães fumantes apresentaram pesos ao nascer significativamente menores do que filhos de mães não fumantes.

As velocidades de incremento médio diário de peso no primeiro e no segundo trimestres, para ambos os sexos, mostram que o crescimento é mais veloz no primeiro trimestre do que no segundo trimestre e que essa redução ocorre independentemente da duração do AME. Isso, provavelmente, expressa o comportamento normal do crescimento, tendo sido descrita, também, em outros estudos ${ }^{1,3,9}$.

Resultados como esses evidenciam que o crescimento nos primeiros seis meses de vida é determinado por diversos fatores e que o aleitamento materno exclusivo dos três aos seis meses confere crescimento adequado; assim, as aparentes alterações no crescimento de crianças em aleitamento exclusivo devem ser avaliadas com cautela, a fim de evitar a introdução desnecessária de alimentação complementar.

Reforçam, também, a importância de ações efetivas voltadas às principais causas do desmame precoce, no intuito de reduzir a alta prevalência de abandono do AME nos primeiros seis meses de vida.

\begin{abstract}
There are controversies about the growth of infants who are exclusively breastfed (EB) from three to six months of life, although the World Health Organization recommends that exclusive breastfeeding (EB) until the sixth month of life assures an adequate growth. Objective: To measure the average velocity of daily increment in weight (VDIW) in the first two life quarters, according to the duration of EB, adjusted for other possible determinants of growth. Methods: a cohort of 347 newborns in a university hospital of São Paulo was studied in the years of 1998 and 1999, using multiple linear regression, and applying hierarchical modeling. Results: $17.5 \%$ of the children were EB at three months; $2.4 \%$ at six months. In the first quarter, among the girls, larger VDIW is related to larger duration of EB. For the boys, VDIW does not differ according to the duration of EB. Children's VDIW in the second quarter of age was not influenced by EB duration. Conclusions: EB assures adequate growth in the first two life quarters; the apparent alterations of the growth of children in EB should be evaluated with caution, in order to avoid the unnecessary introduction of complementary feeding.
\end{abstract}

Key words: Growth. Children Nutrition. Breastfeeding. Linear Regression. 


\section{REFERÊNCIAS}

1. Arifeen SE, Black RE, Caufield LE, Antelman G, Baqui AH. Determinants of infant growth in the slums of Dhaka: size and maturity at birth, breastfeeding and morbidity. Eur. J. Clin Nutr. 2001; 55:167-78.

2. Barros FC, Victora CG, Vaughan JP. Amamentação e dieta In: Epidemiologia das desigualdades: um estudo longitudinal de 6000 crianças brasileiras. São Paulo: Hucitec. 1989; 117-27.

3. Barros Filho AA, Barbieri MA, Santoro JR, Bettiol H. Crescimento de lactentes até os 6 meses de idade alimentados com leite materno e com leite artificial. Medicina, Ribeirão Preto. 1996; 29: 479-487.

4. Diaz S, Herreros C, Avarena R, Casado ME, Reyes MV, Schiappcasse V. Breast-feeding duration and growth of fully breast-fed infants in a poor urban Chilean population. Am J Clin Nutr. 1995; 62 :371-6.

5. Hitchcock NE, Gracy M, Owles EN. Growth of healthy breast-fed infants in first six months. Lancet. 1981; 11: 64-5.

6. Juez G, Díaz S, Casado ME, Durán E, Salvatiera A-M, Peralta O, Croxato HB. Growth pattern of selected urban Chilean infants during exclusive breast -feeding. Am J Clin Nutr.1983; 38: 462-8.

7. Leão M, Coitinho DC, Recine E, Costa LAL, Lacerda AJ. O perfil do aleitamento materno no Brasil. In: Monteiro MFG, Cervini R., organizadores. Perfil estatístico de crianças e mães no Brasil: aspectos de saúde e nutrição das crianças no Brasil. Rio de Janeiro. FIBGE/ UNICEF/INAN; 1989; 97-109.

8. Longo GZ, Souza JMP, Souza SB, Szarfarc SC. Crescimento de crianças até os seis meses de idade segundo categorias de aleitamento materno. Rev. Bras. Saúde Materno Infantil. 2005; 5 Supl 1: 109-118.

9. Marques RFSV, Lopez FA, Braga JAP. O C. O crescimento de crianças alimentadas com leite materno exclusivo nos primeiros 6 meses de vida. J. Pediatria. 2004; 80 Supl 2: S99-105.

10. Martorell R, Khan LK, Schoroeder DG. Reversibility of stunting: epidemiological findings in children from developing countries. Eur J Clin Nutr. 1994; 48 Suppl 1:S45-S57.

11. Mathur S, Mathur GP, Gupta V, Singh YD, Kushwaha KP, Verma A, Rathi AK. Growth patterns in breastfed babies during first six months of life. Indian Pediatric. 1994; 31 Supl 3: 275-8.

12. Murahovschi J, Teruya KM, Nascimento ET, Bueno LLGS, Pinheiro L, Maneta ME et al. Curvas e tabelas de crescimento de lactentes brasileiros de zero a seis meses de idade alimentados exclusivamente com leite materno. J Pediatria. 1987; 63 Supl 4: 1-25.

13. Onis, M. de, Victora, CG. Gráficos de crescimento para bebês alimentados com leite materno. J. Pediatria. 2004, 80(2):85-87. ISSN 0021-7557.

14. Pimentel VAF, Joaquim MCM, Pimentel EF, Garcia DMM. El crescimiento de los niños alimentados exclusivamente com leche materna durante los seis primeiros meses de vida. Bol. Oficina Sanit Panam. 1991; 110:311-8.

15. Posada ME, Rubén M, Esquivel M. On growth of children: effect of some socioeconomic and genetic factors. Acta Paediatr Hung. 1987; 28:37-43.

16. Puccini RF, Wechsler R, Silva EMK, Resegue $R$. Fatores de risco para morbidade e desnutrição em crianças acompanhadas em programa de atenção à saúde da criança. J. Pediatria. 1997; 73 Supl 4:S244-251.

17. Rivera J, Ruel MT. Growth retardation in the first three months of life among rural Guatemala children. Eur J Clin Nutr. 1997; 51:92-6.

18. Seward JK, Serdula MK. Infant feeding and growth: task force on infant-feeding practices. Pediatrics. 1984; 74 Suppl 1: 728-62.

19. Siqueira AAF, Santos JLF, Saqueiro CG, Luz ET, Araújo MCA. Estado Nutricional e hábito de fumar maternos, crescimento intra-uterino e pós-natal. Rev, Saúde Pública. 1985; 19:37-50.

20. Stata Corp. Stata statistical software: Release 8.2 College Station, TX: Stata Corporation, 2003.

21. Victora CG, Huttly AR, Fuchs SC, Olinto MTA. The role of conceptual frameworks in epidemiological analysis: A hierarchical approach. Int. Journ of Epidem. 1997; 26: 224-7.

22. Waterlow JC, Ashworth A, Griffiths M. Faltering infant growth in less-developed countries. Lancet. 1980, v ii:1176-8.

23. Waterlow JC, Thomson AM. Observations on the adequacy of breastfeeding. Lancet. 1979; 2 :238-42. 
24. Whitehead RG, Paul AA. Growth charts and the assessment of infant feeding practices in the western world and in developing countries. Early Hum Dev. 1984; 9: 187-207.

25. World Health Organization (WHO). Expert consultation on the optimal duration of exclusive breastfeeding. Conclusions and recommendations. World Wealth Organization. Document A54/INF.DOC./4, Geneva, 28-30 March 2001.
26. Zeferino AMB, Barros Filho AA, Bettiol H, Barbieri MA. Acompanhamento do crescimento. Jornal de Pediatria 2003;19 Supl.1 :S23-31.

27. WHO Multicentre Growth Reference Study Group. Breastfeeding in the WHO Multicentre Growth Reference Study. Acta Paediatr. 2006; Suppl 450: 16-26.

Recebido em 13/12/2006 Modificado em 19/04/2007 Aprovado em 24/04/2007 